\title{
Engineering Escherichia coli for high-yielding hydroxytyrosol synthesis from biobased L-tyrosine
}

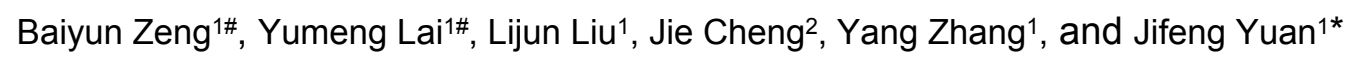

1 State Key Laboratory of Cellular Stress Biology, Innovation Center for Cell Signaling Network, School of Life Sciences, Xiamen University, Fujian, PR China, 361102

2 State Key Laboratory of Marine Environmental Science, College of Ocean and Earth Sciences, Xiamen University, Fujian, PR China, 361102

\# These authors contribute equally to the work.

* $\quad$ Corresponding author address: State Key Laboratory of Cellular Stress Biology, Innovation Center for Cell Signaling Network, School of Life Sciences, Xiamen University, Fujian, PR China, 361102. Email: jfyuan@xmu.edu.cn (J. Yuan) 


\section{Chemicals, Biochemicals, and Medium}

All chemicals were obtained commercially and used without further purification: Chemicals purchased from Sigma-Aldrich: L-tyrosine ( $\geq 98 \%)$, D-glucose $(99 \%)$.

Chemicals purchased from Tokyo Chemical Industry Co., LTD: dopamine (>98\%), hydroxytyrosol (>98\%)

Chemicals purchased from Inalco: kanamycin sulfate salt $(>75 \%)$, ampicillin sodium salt $(>84.5 \%)$ chloramphenicol sulfate salt $(>85 \%)$.

Chemicals purchased from CNW Technologies: acetonitrile (HPLC, $\geq 99.9 \%$ ).

Chemicals purchased from Sinopharm Chemical Reagent: methanol $(>99.8 \%)$, ethanol absolute $(>99.7 \%)$, glycerol $(>99 \%), \mathrm{HCl}$ (37\%), $\mathrm{NaCl}(\geq 99.5 \%), \mathrm{K}_{2} \mathrm{HPO}_{4} \cdot 3 \mathrm{H}_{2} \mathrm{O}(\geq 99 \%), \mathrm{KH}_{2} \mathrm{PO}_{4}(\geq 99.5 \%), \mathrm{NH}_{4} \mathrm{Cl}(\geq 99.5 \%), \mathrm{CaCl}_{2}(\geq 96 \%)$.

Biochemicals and kits were purchased from commercial suppliers:

Inalco: Isopropyl $\beta$-D-1-thiogalactopyranoside (IPTG, >99\%).

Sangon Biotech: LB medium, tryptone, yeast extract and agar.

Biomer Technology: plasmid miniprep kit, gel extraction kit, DNA purification kit, PCR purification kit.

New England Biolabs: restriction enzymes, Phusion High-Fidelity DNA Polymerase and T4 ligase.

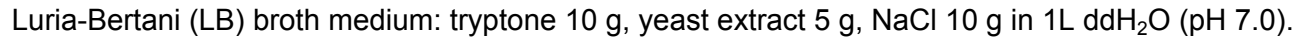

Terrific Broth (TB) medium containing $\mathrm{K}_{2} \mathrm{HPO}_{4} \cdot 3 \mathrm{H}_{2} \mathrm{O}\left(12.54 \mathrm{~g} \mathrm{~L}^{-1}\right), \mathrm{KH}_{2} \mathrm{PO}_{4}\left(2.32 \mathrm{~g} \mathrm{~L}^{-1}\right)$, tryptone $\left(12 \mathrm{~g} \mathrm{~L}^{-1}\right)$, glycerol $(0.4 \%)$ and yeast extract $\left(24 \mathrm{~g} \mathrm{~L}^{-1}\right)$ was used for culturing the $E$. coli cells $(100-200 \mathrm{~mL})$ for biotransformation.

\section{Gene Sequences}

$H p a B C$ gene was amplified from genomic DNA of Escherichia coli. The DNA sequence is:

ATGAAACCAGAAGATTTCCGCGCCAGTACCCAACGTCCGTTCACCGGGGAAGAGTATCTGAAAAGCCTGCAGGATGGTCG CGAGATCTATATCTATGGCGAGCGAGTGAAAGACGTCACTACTCATCCGGCATTTCGTAATGCGGCAGCGTCTGTTGCCCA ACTGTACGACGCGCTGCACAAACCGGAGATGCAGGACTCTCTGTGTTGGAACACCGACACCGGCAGCGGCGGCTATACC CATAAATTCTTCCGCGTGGCGAAAAGTGCCGACGACCTGCGCCAGCAACGCGACGCCATCGCTGAGTGGTCACGCCTGAG CTATGGCTGGATGGGCCGTACCCCAGACTACAAAGCTGCTTTCGGTTGCGCACTGGGCGCGAATCCGGGCTTTTACGGTC AGTTCGAGCAGAACGCCCGTAACTGGTATACCCGTATTCAGGAAACTGGCCTCTACTTTAACCACGCGATTGTTAACCCAC CGATCGATCGTCATTTGCCGACCGATAAAGTGAAAGACGTTTACATCAAGCTGGAAAAAGAGACTGACGCCGGGATTATCG TTAGCGGTGCGAAAGTGGTTGCCACCAACTCGGCGCTGACTCACTACAACATGGTTGGCTTCGGCTCGGCACAAGTAATG GGCGAAAACCCGGACTTCGCGCTGATGTTCGTTGCGCCAATGGATGCTGATGGCGTGAAATTAATCTCCCGCGCCTCTTAT GAGATGGTCGCGGGTGCTACCGGCTCACCGTATGACTACCCGCTCTCCAGCCGCTTCGATGAGAATGATGCGATTCTGGT GATGGATAACGTGCTGATCCCATGGGAAAACGTGCTGATCTACCGCGATTTTGATCGCTGCCGTCGCTGGACGATGGAAC GCGGTTTTGCCCGTATGTATCCGCTGCAAGCCTGTGTGCGCCTGGCAGTGAAACTCGACTTCATTACGGCACTGCTGAAAA AATCACTCGAATGTACCGGCACCCTGGAGTTCCGTGGTGTGCAGGCCGATCTCGGTGAAGTGGTGGCGTGGCGCAACAC CTTCTGGGCATTGAGTGACTCGATGTGTTCTGAAGCGACGCCGTGGGTCAACGGGGCTTATTTACCGGATCATGCCGCAC TGCAAACCTATCGCGTACTGGCACCAATGGCCTACGCGAAGATCAAAAACATTATCGAACGCAACGTTACCAGTGGCCTGA TCTACCTCCCTTCCAGTGCCCGTGACCTGAACAATCCGCAGATCGACCAGTATCTGGCGAAGTATGTGCGCGGTTCGAATG GTATGGATCACGTCCAGCGCATCAAGATCCTCAAACTGATGTGGGACGCCATTGGCAGCGAGTTTGGTGGTCGCCACGAA CTGTATGAAATCAACTACTCCGGTAGCCAGGATGAGATTCGCCTGCAGTGTCTGCGCCAGGCACAAAGCTCCGGCAATATG GACAAGATGATGGCGATGGTTGATCGCTGCCTGTCGGAATACGACCAGAACGGCTGGACTGTGCCGCACCTGCACAACAA CGACGATATCAACATGCTGGATAAGCTGCTGAAATAACGCAGCAGGAGGTTAAGATGCAATTAGATGAACAACGCCTGCGC TTTCGTGACGCGATGGCCAGCCTGTCGGCAGCGGTAAATATTATCACCACCGAGGGCGACACCGGACAATGCGGGATTAC GGCAACGGCTGTCTGCTCGGTCACGGATACACCACCGTCGCTGATGGTGTGCATTAACGCCAACAGTGCGATGAACCCGG TTTTTCAGGGCAACGGCAAGTTGTGCGTCAACGTCCTCAACCATGAGCAGGAACTGATGGCACGCCACTTCGCGGGCATG ACAGGCATGGCGATGGAAGAGCGTTTTAGCCTCTCATGCTGGCAAAAAGGTCCGCTGGCGCAGCCGGTGCTAAAAGGTTC GCTGGCCAGTCTTGAAGGTGAGATCCGCGATGTGCAGGCAATTGGCACACATCTGGTGTATCTGGTGGAGATTAAAAACAT CATCCTCAGTGCAGAAGGTCACGGACTTATCTACTTTAAACGCCGTTTCCATCCGGTGATGCTGGAAATGGAAGCTGCGAT TTAA

MAO gene from Micrococcus luteus was codon optimized for $E$. coli and synthesized by GenScript. The optimized DNA sequence is:

ATGAGCAATCCGCACGTTGTGATTGTGGGTGCAGGTTTTGCGGGTCTGGTTGCGGCTCGTGAACTGCAAATGGCGGGTGT GGATGTTGAAATCGTGGAAGCGCGTGACCGTGTTGGTGGTCGTGCTTGGACCGAAGAACGTATGGGTCGTCCGCTGGAAC TGGGTGCTACCTGGGTGCATTGGATGCAACCGCACGTTTGGAGCGAAATTACCCGTTACGATCAGAGCATCTATCCGAGC CCGTTTTGCGATGACGCGTACTGGATTACCGGTGGTCGTGTTGAACACGGTACCGAAGCTGACCTGGATGCAGCGCTGGC TCGTCCGATGGCAAAAATCTTCGAAGATAGCCGTGAATTTTTCCCGTACCCGTATGAACCGCTGCATGTGCTGGACGAAAG CAGCGGTAGCACCCCGGAACTGCGTGAACGTTTTCGTGCTGCAGACCAAGGTAGCGTTCTGGATTGCCTGAAGGGTGGTG 
ACTTCACCCAGGAAGAACGTGACCTGTGTGATGCATACTGGAGCGCGGCTTATATTGGTGACCCGCATCAAGGTAGCCCG CTGATGGCGAAACAGTGGGCAGCGCTGAGCGATCACCGTCTGAGCCTGGTGGACGAACAAACCCTGCGTTTTAAGCTGAC CCATGGTATGCGTGGTCTGTACGAAAATATTGCTGCAGACCTGCGTTGCCCGATCCGTCTGAACACCCCGGTGACCGCAG TTGACCATCGTAGCGATGGTGCGACCGTTACCCTGGGTACCGGTGAAAAGATTAGCTGTGATAGCGTGATCGTTACCGTGC CGGTTGGTGCGCTGCCGACCATTGAGTTTACCCCGGGTCTGCCAAGCGGTATGAGAACCGTGATCGACCAGCGTTGGAAT AGCACCGGTTGCAAAATTTGGGTGAAAGTTAAGGGTCATCACAGCATCTTGGGTTATGCTCCAACCCCGCACAAGGCTGCT GTTTTTCGTAGCGAATTTTTCATGGATGACGATACCACCATCTGTGTGGGTTTCGGTAGCCATCACGACGCTGTTGATCTGA CCGACCCGCGTGATGCACAAGCGATTGTGGACCAGTGGCGCCCGGACCTGGAAGTTGTGGATTGTACCGGTCATGACTG GGTTGCTGATCGTTGGAGCGGTCAAGCTTGGGCAACCCTGCGTAGCGGCCAGTTTACCAACGGTTGGCATCACTTCCGTA GCACCGATAGCCGTCTGCGTTTTGCTGGTGCTGACTGGGCTCGTGGTTGGCGTGGTGTTGTGGTTGATGGTGCTATTGAA ACCGGTCTGAGCACCGCACGTGACGTTCTGCGTGATATCCGTGCATAA

DODC gene was amplified from genomic DNA of Pseudomonas putida KT2440. The DNA sequence is:

ATGACCCCCGAACAATTCCGCCAGTACGGCCACCAACTGATCGACCTGATTGCCGACTACCGCCAGACCGTGGGCGAACG CCCGGTCATGGCCCAGGTCGAACCTGGCTATCTCAAGGCCGCCTTGCCCGCAACTGCCCCTCAACAAGGCGAACCTTTCG CGGCCATTCTCGACGACGTCAATAACCTGGTCATGCCCGGCCTGTCCCATTGGCAGCACCCGGACTTCTATGGCTATTTCC CTTCCAATGGCACCCTGTCCTCGGTGCTGGGGGACTTCCTCAGTACCGGTCTGGGCGTGCTGGGCCTGTCCTGGCAATCC AGCCCGGCCCTGAGCGAACTGGAAGAAACCACCCTCGACTGGCTGCGCCAGTTGCTTGGCCTGTCTGGCCAGTGGAGTG GGGTGATCCAGGACACTGCCTCGACCAGCACCCTGGTGGCGCTGATCAGTGCCCGTGAACGCGCCACTGACTACGCCCT GGTACGTGGTGGCCTGCAGGCCGAGCCCAAGCCTTTGATCGTGTATGTCAGCGCCCACGCCCACAGCTCGGTGGACAAG GCTGCACTGCTGGCAGGTTTTGGCCGCGACAATATCCGCCTGATTCCCACCGACGAACGCTACGCCCTGCGCCCAGAGG CACTGCAGGCGGCGATCGAACAGGACCTGGCTGCCGGCAACCAGCCGTGCGCCGTGGTTGCCACCACCGGCACCACGA CGACCACTGCCCTCGACCCGCTGCGCCCGGTCGGTGAAATCGCCCAGGCCAATGGGCTGTGGTTGCACGTTGACTCGGC CATGGCCGGTTCGGCGATGATCCTGCCCGAGTGCCGCTGGATGTGGGACGGCATCGAGCTGGCCGATTCGGTGGTGGTC AACGCGCACAAATGGCTGGGTGTGGCCTTCGATTGCTCGATCTACTACGTGCGCGATCCGCAACACCTGATCCGGGTGAT GAGCACCAATCCCAGCTACCTGCAGTCGGCGGTGGATGGCGAGGTGAAGAACCTGCGCGACTGGGGGATACCGCTGGGC CGTCGGTTCCGTGCGTTGAAGCTGTGGTTCATGTTGCGCAGCGAGGGTGTCGACGCATTGCAGGCGCGGCTGCGGCGTG ACCTGGACAATGCCCAGTGGCTGGCGGGGCAGGTCGAGGCGGCGGCGGAGTGGGAAGTGTTGGCGCCAGTACAGCTGC AAACCTTGTGCATTCGCCATCGACCGGCGGGGCTTGAAGGGGAGGCGCTGGATGCGCATACCAAGGGCTGGGCCGAGCG GCTGAATGCATCCGGCGCTGCTTATGTGACGCCGGCTACACTGGACGGGCGGTGGATGGTGCGGGTTTCGATTGGTGCG CTGCCGACCGAGCGGGGGGATGTGCAGCGGCTGTGGGCACGTCTGCAGGACGTGATCAAGGGCTGA

PAR gene from Solanum lycopersicum was codon optimized for Escherichia coli. The optimized sequence is:

ATGAGCGTGACCGCGAAAACCGTGTGTGTTACCGGCGCCAGCGGCTACATCGCCTCTTGGCTTGTAAAGTTTCTGTTGCAC TCGGGTTATAACGTTAAAGCGTCAGTCCGCGATCCGAACGACCCGAAAAAAACGCAGCACCTGCTGTCTCTGGGCGGCGC GAAAGAACGGCTGCACCTGTTCAAAGCGAATCTGCTGGAAGAAGGTTCGTTCGATGCGGTTGTTGACGGTTGCGAAGGTG TGTTCCATACCGCGTCCCCTTTCTACTATTCTGTAACCGATCCGCAGGCCGAGCTTCTGGATCCGGCAGTAAAAGGCACTC TGAACCTGCTCGGTTCCTGTGCTAAAGCGCCTTCAGTTAAGCGTGTAGTGCTCACCAGCAGCATCGCTGCCGTTGCGTACT CTGGTCAACCACGTACTCCTGAAGTAGTAGTGGATGAATCGTGGTGGACGTCTCCTGATTATTGTAAAGAAAAACAGCTGT GGTACGTCTTAAGTAAAACGCTGGCGGAAGATGCGGCTTGGAAATTTGTGAAAGAAAAGGGGATTGACATGGTCGTTGTAA ATCCTGCAATGGTCATTGGACCGTTACTGCAGCCAACCCTCAATACGAGCAGCGCCGCGGTGCTGTCTCTTGTAAACGGG GCGGAAACATATCCTAATAGCTCTTTCGGCTGGGTTAATGTCAAAGATGTGGCGAATGCCCACATTCTTGCCTTTGAAAACC CAAGCGCCAACGGCCGTTACTTGATGGTTGAACGTGTTGCGCACTATAGCGACATCCTGAAAATCCTCCGCGACCTGTACC CTACTATGCAATTGCCTGAAAAATGTGCAGATGATAACCCGCTGATGCAGAATTACCAGGTCTCAAAAGAAAAAGCGAAATC ACTGGGCATCGAATTTACTACTCTTGAGGAAAGTATTAAAGAAACAGTAGAATCCTTGAAAGAAAAAAAATTTTTCGGCGGG AGTTCAAGCATGTGA

LAAD gene from Proteus mirabilis was codon optimized for $E$. coli and synthesized by GenScript. The optimized DNA sequence is:

ATGAACATTTCACGTCGCAAGCTGCTGCTGGGTGTTGGTGCTGCTGGTGTGTTGGCGGGTGGTGCAGCTCTGGTTCCAAT GGTGCGTCGTGATGGTAAATTTGTTGAAGCAAAGAGCCGTGCGAGCTTCGTGGAAGGTACCCAAGGTGCGCTGCCGAAAG AAGCTGACGTTGTGATTATCGGTGCTGGTATTCAGGGTATCATGACCGCTATTAATCTGGCAGAACGTGGTATGAGCGTTA CCATTCTGGAAAAGGGTCAAATCGCAGGTGAACAGAGCGGTCGTGCGTACAGCCAAATTATCAGCTATCAGACCAGCCCG GAAATTTTTCCGCTGCATCACTACGGTAAAATCCTGTGGCGTGGTATGAACGAAAAGATTGGTGCGGATACCAGCTATCGT ACCCAAGGTCGTGTTGAAGCTCTGGCAGATGAAAAAGCACTGGACAAGGCGCAGGCTTGGATCAAAACCGCGAAGGAAGC TGCAGGTTTTGACACCCCGCTGAATACCCGTATTATCAAAGGTGAAGAACTGAGCAACCGTCTGGTTGGTGCTCAAACCCC GTGGACCGTGGCTGCTTTCGAAGAAGATAGCGGTAGCGTTGACCCGGAAACCGGTACCCCGGCACTGGCTCGTTACGCTA AACAGATTGGTGTTAAGATCTATACCAACTGCGCTGTGCGTGGTATTGAAACCGCGGGTGGTAAAATCAGCGATGTTGTGA GCGAAAAAGGTGCGATCAAGACCAGCCAAGTGGTGCTGGCGGGTGGTATTTGGAGCCGTCTGTTTATGGGTAATATGGGT ATTGACATCCCGACCCTGAACGTTTACCTGAGCCAACAACGTGTTAGCGGTGTGCCAGGTGCGCCGCGTGGTAATGTGCA TCTGCCGAACGGTATCCACTTTCGTGAACAAGCTGATGGTACCTATGCTGTTGCACCGCGTATTTTCACCAGCAGCATCGT GAAAGACAGCTTTCTGCTGGGTCCGAAGTTCATGCATCTGCTGGGTGGTGGTGAACTGCCGCTGGAATTTTCTATCGGTGA AGACCTGTTTAATAGCTTCAAAATGCCGACCAGCTGGAACCTGGACGAAAAGACCCCGTTTGAACAATTCCGTGTTGCGAC CGCTACCCAAAATACCCAGCACCTGGATGCAGTTTTTCAGCGTATGAAAACCGAATTTCCGGTGTTCGAAAAGAGCGAAGT TGTGGAACGTTGGGGTGCTGTTGTGAGCCCGACCTTCGACGAACTGCCGATTATCAGCGAAGTTAAGGAATACCCGGGTC 
TGGTTATTAACACCGCTACCGTGTGGGGTATGACCGAAGGTCCGGCAGCGGGTGAAGTTACCGCAGATATTGTGATGGGT AAAAAGCCGGTTATTGATCCGACCCCGTTTAGTTTGGATCGTTTTAAGAAGTAA

AR010 was amplified from genomic DNA of Saccharomyces cerevisiae. The DNA sequence is:

ATGGCACCTGTTACAATTGAAAAGTTCGTAAATCAAGAAGAACGACACCTTGTTTCCAACCGATCAGCAACAATTCCGTTTG GTGAATACATATTTAAAAGATTGTTGTCCATCGATACGAAATCAGTTTTCGGTGTTCCTGGTGACTTCAACTTATCTCTATTA GAATATCTCTATTCACCTAGTGTTGAATCAGCTGGCCTAAGATGGGTCGGCACGTGTAATGAACTGAACGCCGCTTATGCG GCCGACGGATATTCCCGTTACTCTAATAAGATTGGCTGTTTAATAACCACGTATGGCGTTGGTGAATTAAGCGCCTTGAACG GTATAGCCGGTTCGTTCGCTGAAAATGTCAAAGTTTTGCACATTGTTGGTGTGGCCAAGTCCATAGATTCGCGTTCAAGTAA CTTTAGTGATCGGAACCTACATCATTTGGTCCCACAGCTACATGATTCAAATTTTAAAGGGCCAAATCATAAAGTATATCATG ATATGGTAAAAGATAGAGTCGCTTGCTCGGTAGCCTACTTGGAGGATATTGAAACTGCATGTGACCAAGTCGATAATGTTAT CCGCGATATTTACAAGTATTCTAAACCTGGTTATATTTTTGTTCCTGCAGATTTTGCGGATATGTCTGTTACATGTGATAATTT GGTTAATGTTCCACGTATATCTCAACAAGATTGTATAGTATACCCTTCTGAAAACCAATTGTCTGACATAATCAACAAGATTA CTAGTTGGATATATTCCAGTAAAACACCTGCGATCCTTGGAGACGTACTGACTGATAGGTATGGTGTGAGTAACTTTTTGAA CAAGCTTATCTGCAAAACTGGGATTTGGAATTTTTCCACTGTTATGGGAAAATCTGTAATTGATGAGTCAAACCCAACTTATA TGGGTCAATATAATGGTAAAGAAGGTTTAAAACAAGTCTATGAACATTTTGAACTGTGCGACTTGGTCTTGCATTTTGGAGTC GACATCAATGAAATTAATAATGGGCATTATACTTTTACTTATAAACCAAATGCTAAAATCATTCAATTTCATCCGAATTATATTC GCCTTGTGGACACTAGGCAGGGCAATGAGCAAATGTTCAAAGGAATCAATTTTGCCCCTATTTTAAAAGAACTATACAAGCG CATTGACGTTTCTAAACTTTCTTTGCAATATGATTCAAATGTAACTCAATATACGAACGAAACAATGCGGTTAGAAGATCCTA CCAATGGACAATCAAGCATTATTACACAAGTTCACTTACAAAAGACGATGCCTAAATTTTTGAACCCTGGTGATGTTGTCGTT TGTGAAACAGGCTCTTTTCAATTCTCTGTTCGTGATTTCGCGTTTCCTTCGCAATTAAAATATATATCGCAAGGATTTTTCCTT TCCATTGGCATGGCCCTTCCTGCCGCCCTAGGTGTTGGAATTGCCATGCAAGACCACTCAAACGCTCACATCAATGGTGGC AACGTAAAAGAGGACTATAAGCCAAGATTAATTTTGTTTGAAGGTGACGGTGCAGCACAGATGACAATCCAAGAACTGAGC ACCATTCTGAAGTGCAATATTCCACTAGAAGTTATCATTTGGAACAATAACGGCTACACTATTGAAAGAGCCATCATGGGCC CTACCAGGTCGTATAACGACGTTATGTCTTGGAAATGGACCAAACTATTTGAAGCATTCGGAGACTTCGACGGAAAGTATAC TAATAGCACTCTCATTCAATGTCCCTCTAAATTAGCACTGAAATTGGAGGAGCTTAAGAATTCAAACAAAAGAAGCGGGATA GAACTTTTAGAAGTCAAATTAGGCGAATTGGATTTCCCCGAACAGCTAAAGTGCATGGTTGAAGCAGCGGCACTTAAAAGAA ATAAAAAATAG 


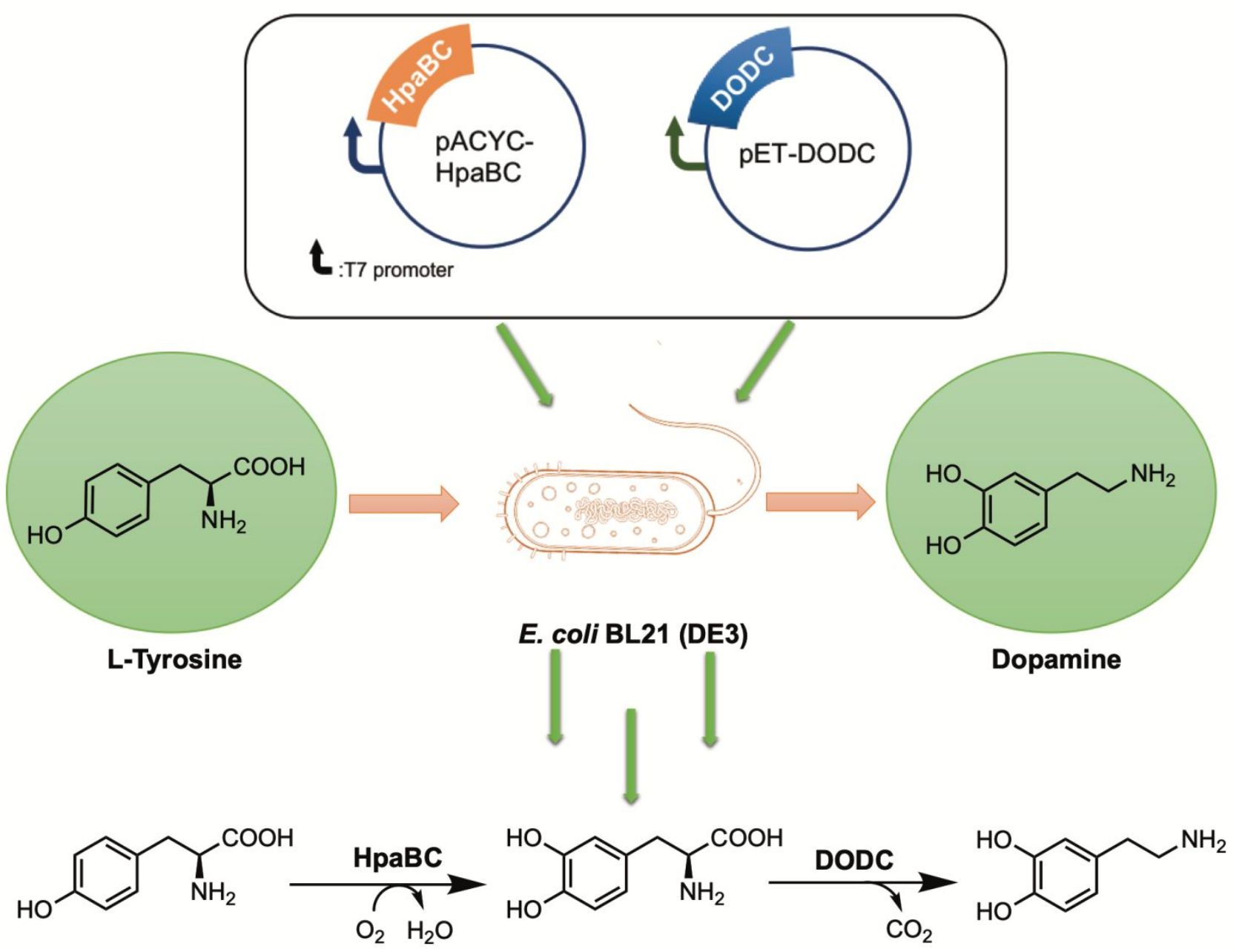

Figure S1. Enzymatic synthesis of dopamine comprising the native hydroxylase (HpaBC) from E. coli and L-DOPA decarboxylase (DODC) from P. putida KT2440. pACYC-HpaBC and pET-DODC were constructed and transformed into E. coli BL21 (DE3) strain. 
A

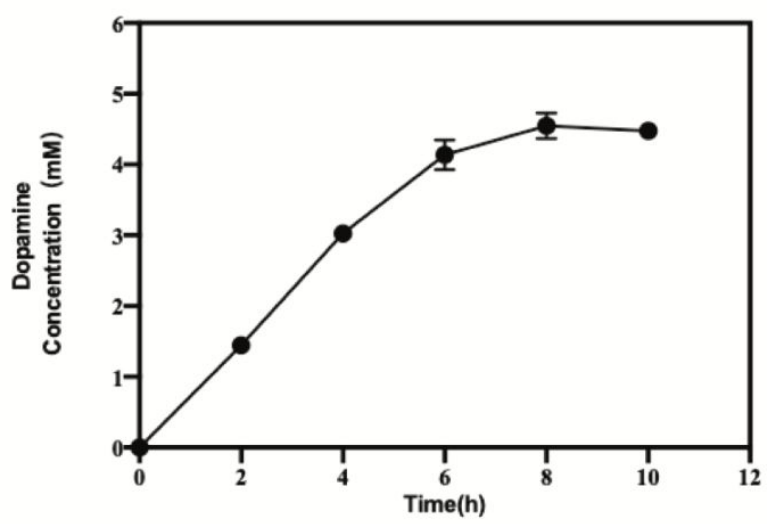

B

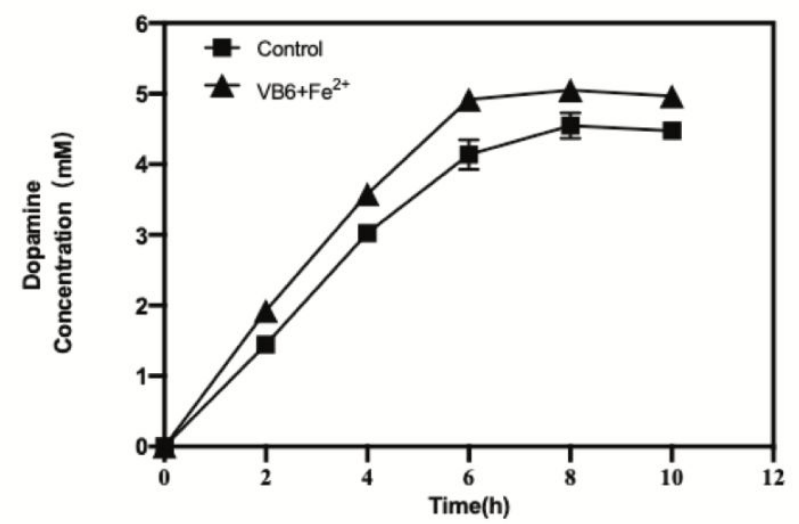

Figure S2. Biotransformation of L-tyrosine into dopamine. (A) Time course of biotransformation of L-tyrosine (5 mM) to dopamine with resting cells of $E$. coli BL21 (DE3) cells (10 g-cdw/L). (B) Compare dopamine production with the presence and absence of $50 \mu \mathrm{M}$ vitamin $\mathrm{B} 6$ and $200 \mu \mathrm{M} \mathrm{Fe}{ }^{2+}$. All biocatalytic experiments were performed in $1 \mathrm{~mL} \mathrm{KP}$ buffer $(200 \mathrm{mM}, \mathrm{pH} 8.0,2 \%$ glucose $)$ at $30^{\circ} \mathrm{C}$ for $10 \mathrm{~h}$. Experiments were performed in triplicate, and the data represent the mean value with standard deviations. 

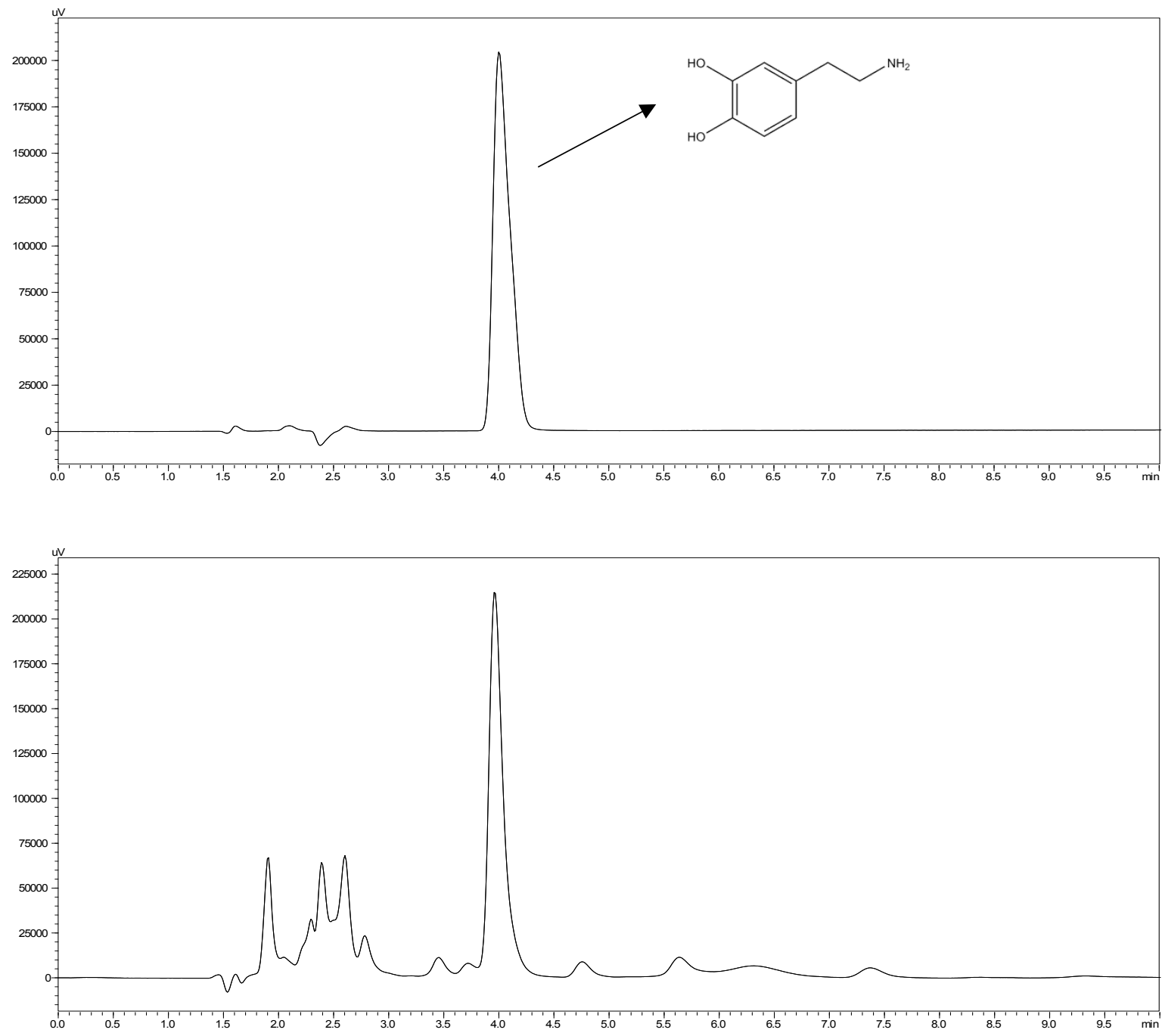

Figure S3. HPLC chromatograms of dopamine. Authentic dopamine standard (top); Sample from biotransformation of L-tyrosine (5 mM) with E. coli BL21 (DE3) (10 g-cdw/L) in KP buffer (200 mM, pH 8.0) with $2 \%$ glucose (bottom). 

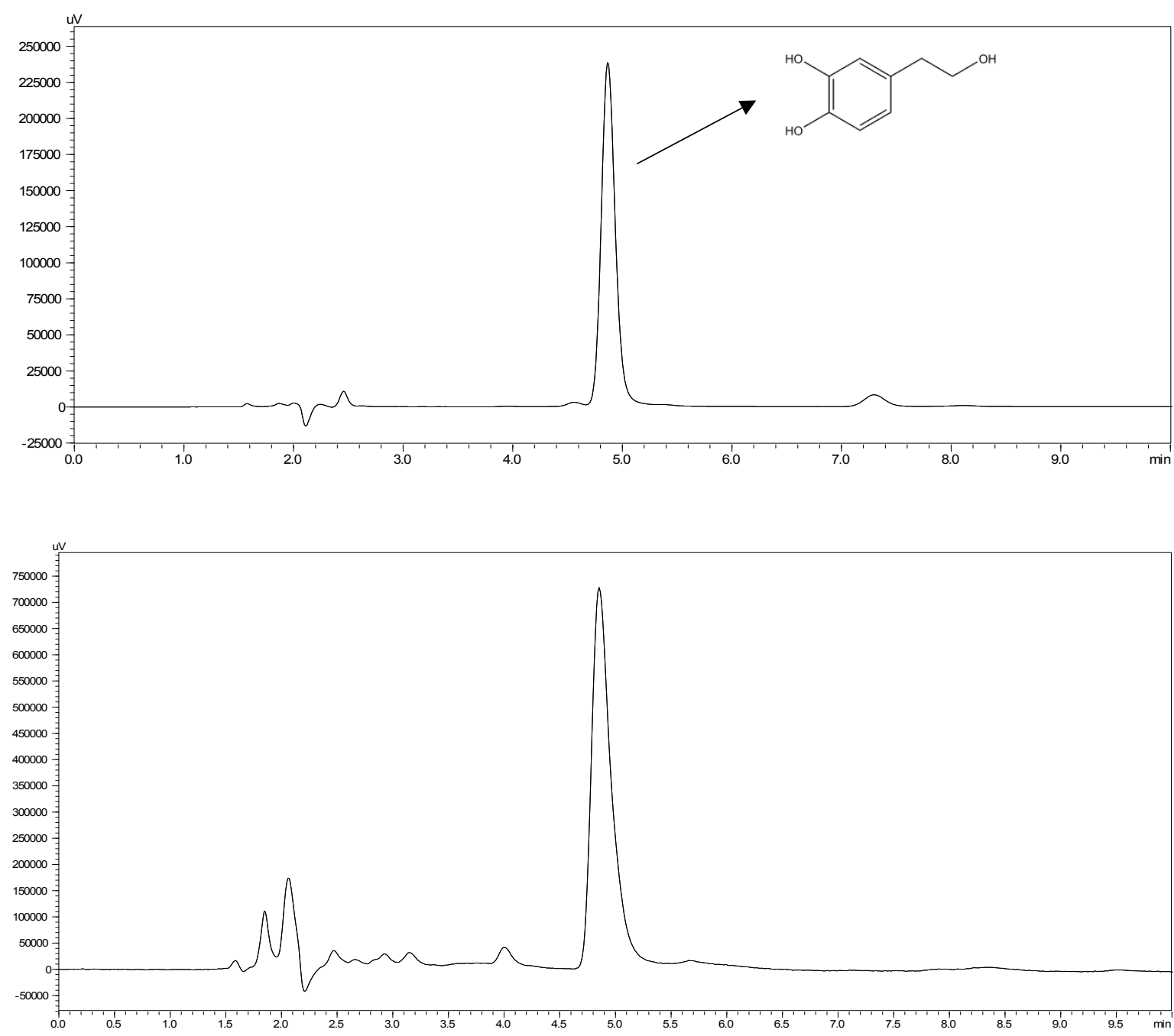

Figure S4. HPLC chromatograms of hydroxytyrosol (HT). Authentic HT standard (Top); Sample from biotransformation of Ltyrosine (25 mM) with E. coli BL21 (DE3) (10 g-cdw/L) in KP buffer (200 mM, pH 8.0) with 2\% glucose (bottom). 
Table S1. List of primers used in this study

\begin{tabular}{ll}
\hline Name of primers & Sequence (the restriction enzyme digestion sites are underlined) \\
\hline HpaBC_BamHIffwd & CGGGATCCGATGAAACAGAAGATTTCC \\
HpaBC_Xhol_rev & AGAGACTCGAGTTAAATCGCAGCTTCCATTTC \\
DODC_Esp3I_fwd & TTCGTCTCGGATCCGATGACCCCCGAACAATTCCG \\
DODC_Esp3I_rev & TTCGTCTCACTCCTCAGCCCTTGATCACGTCC \\
MAO_Esp3I_fwd & TTCGTCTCCAGGAGATATATTATGAGCAATCCGCACGTTG \\
MAO_Esp3I_rev & TTCGTCTCCTCGAGTTATGCACGGATATCACGC \\
DODC_Xhol_rev & AGAGACTCGAGTCAGCCCTTGATCACGTCC \\
LAAD_Bsal_fwd & TTGGTCTCGGATCCGATGAACATTTCACGTCGCAAGC \\
LAAD_Bsal_rev & TTGGTCTCATCCTTTACTTCTTAAAACGATCCAAAC \\
ARO10_Bsal_fwd & TTGGTCTCAAGGAGATATATAATGGCACCTGTTACAATTG \\
ARO10_Bsal_rev & TTGGTCTCCTCGAGCTATTTTTTATTTCTTTTAAGTG \\
PAR_HindIII_fwd & AGAGAAAGCTTAAGGAGATATATAATGAGCGTGACCGCGAAAAC \\
PAR_Xhol_rev & AGAGACTCGAGTCACATGCTTGAACTCCCG \\
\hline
\end{tabular}


Table S2. List of plasmids and strains used in this study

\begin{tabular}{|c|c|c|}
\hline Name & Detail & Reference \\
\hline pETDuet-1 & T7 expression vector & Novagen \\
\hline pRSFDuet-1 & T7 expression vector & Novagen \\
\hline pACYCDuet-1 & T7 expression vector & Novagen \\
\hline $\begin{array}{l}\text { pET-LAAD- } \\
\text { ARO10 }\end{array}$ & pETDuet-1 derivative with $L A A D$ and $A R 010$ gene & This study \\
\hline pACYC-HраBC & pACYCDuet-1 derivative with $H p a B C$ gene & This study \\
\hline pRSF-PAR & pRSFDuet-1 derivative with PAR gene & This study \\
\hline pET-DODC & pETDuet-1 derivative with $D O D C$ gene & This study \\
\hline pET-DODC-MAO & pETDuet-1 derivative with $D O D C$ and $M A O$ gene & This study \\
\hline E. coli TOP10 & For cloning purpose & Invitrogen \\
\hline E. coli (Dopamine) & E. coli BL21(DE3) with plasmid pACYC-HpaBC and pET-DODC & This study \\
\hline E. coli (HT01) & $\begin{array}{l}\text { E. coli BL21 (DE3) with plasmid pACYC-HpaBC, pET-DODC-MAO and pRSF- } \\
\text { PAR }\end{array}$ & This study \\
\hline E. coli (HT02) & $\begin{array}{l}\text { E. coli BL21 (DE3) with plasmid pET-LAAD-ARO10, pACYC-HpaBC and } \\
\text { pRSF-PAR }\end{array}$ & This study \\
\hline
\end{tabular}

